\title{
El aprendizaje de los estudiantes de enfermería en interacción con el docente experto ${ }^{\dagger}$
}

\author{
Nursing student learning in interaction with the expert teacher \\ Aprendizagem do aluno de enfermagem em interação com o professor especialista
}

\author{
Juliana Jaramillo-Vargas ${ }^{1}$ \\ Natalia Ossa-Garcés ${ }^{2}$ \\ Marcela Carrillo-Pineda ${ }^{3}$
}

\section{Resumen}

Objetivo: Describir el proceso de aprendizaje de los estudiantes de enfermería en la interacción con el docente experto. Materiales y Métodos: Investigación cualitativa, utilizando herramientas de la etnografía. Se identificó un docente reconocido como docente experto. La recolección de la información se hizo en dos fases; en la primera se realizaron observaciones durante el desarrollo de dos clases y 23 entrevistas think aloud a 6 estudiantes. En la segunda fase se realizaron 8 entrevistas a profundidad a seis estudiantes. Se realizó el análisis descriptivo y la categorización en el programa Atlas Ti. Resultados: A Partir de la interacción estudiante-docente emergieron dos categorías que describen el proceso de aprendizaje. La primera es "la interacción entre los estudiantes y el docente experto: un proceso de dos", que se desarrolla mediante la explicación, las intervenciones espontáneas de los estudiantes y los ejercicios de recapitulación. La segunda trata sobre "los aprendizajes: se quedan para la vida", los cuales se evidencian en la identificación de las diversas formas de aprender, los aprendizajes para aprender y los aprendizajes implícitos. Conclusiones: El proceso de aprendizaje en la interacción entre el docente experto y los estudiantes es dinámico, flexible y abierto. Es un espacio donde los estudiantes y el docente construyen una relación activa, propositiva y bidireccional que favorece los procesos formativos en Enfermería, haciendo que los estudiantes obtengan aprendizajes significativos.

Palabras claves: Educación en Enfermería; Estudiante de Enfermería; Docentes de Enfermería; Aprendizaje.

\begin{abstract}
Autor de correspondencia*
1* Enfermera, Universidad de Antioquia. Medellín. Colombia. Recién egresada -Joven Investigadora de la Universidad de Antioquia. Correo: juliana.jaramillov@udea. edu.co (iD)

${ }^{2}$ Enfermera, Universidad de Antioquia. Medellín, Colombia. Recién egresada- Joven Investigadora de la Universidad de Antioquia. Correo: natalia.ossag@udea. edu.co (iD)

${ }^{3}$ Enfermera, Magister en Salud Colectiva. Doctora en Educación. Docente Facultad de Enfermería, Universidad de Antioquia. Medellín, Colombia. Correo: marcela.carrillo@udea.edu.co iD
\end{abstract}

(C) Universidad Francisco de Paula Santander Este es un artículo bajo la licencia CC-BY-NC-ND (c) (i) $(9)$ $\dagger \quad$ Proyecto financiado por el Comité para el Desarrollo de la Investigación - CODI y la Facultad de Enfermería de la Universidad de Antioquia, a
través de la Convocatoria para el desarrollo de proyectos de investigación por estudiantes de pregrado 2018

Recibido: 6 abril 2020. Aprobado: 6 julio 2020.

Para citar este artículo/ To reference this article/ Para citar este artigo/

Jaramillo-Vargas J, Ossa-Garcés N, Carrillo-Pineda M. El aprendizaje de los estudiantes de enfermería en interacción con el docente experto. Rev. cienc. cuidad. 2020; 17(3):61-74. https://doi.org/10.22463/17949831.2269 
ISSN-PRINT

$1794-9831$

E-ISSN 2322-7028

Vol. 17 No. 3

Sep - Dic 2020

Cúcuta, Colombia

\begin{abstract}
Objective: To describe the learning process of nursing students in the interaction with the expert teacher. Materials and Methods: Qualitative research, using tools of ethnography. A teacher recognized as an expert teacher was identified. The information gathering was done in two phases. In the first, observations were made during two classes and 23 think aloud interviews with 6 students. In the second phase, 8 in-depth interviews were conducted with six students. Descriptive analysis and categorization were carried out in the Atlas Ti program. Results: From the student-teacher interaction, two categories emerged that describe the learning process. The first is "the interaction between the students and the expert teacher: a process of two", which is developed through explanation, spontaneous interventions by students and recapitulation exercises. The second deals with "learning: they stay for life", which is evidenced in the identification of the various ways of learning, learning to learn and implicit learning. Conclusions: The learning process in the interaction between the expert teacher and the students is dynamic, flexible and open. It is a space where the students and the teacher build an active, purposeful and bidirectional relationship that favors the educational processes in Nursing, making the students obtain significant learning.
\end{abstract}

Keywords: Nursing Education; Nursing student; Nursing teachers; Learning.

\title{
Resumo
}

Objetivo: Descrever o processo de aprendizagem dos estudantes de enfermagem na interação com o professor especialista. Materiais e métodos: Pesquisa qualitativa, utilizando ferramentas da etnografia. Um educador foi identificado como professor especialista. A coleta de informações foi realizada em duas fases. Na primeira, realizaram-se observações de duas aulas e 23 entrevistas think aloud com 6 alunos. Na segunda fase, foram realizadas oito entrevistas em profundidade com seis alunos. A análise descritiva e a categorização foram realizadas no programa Atlas Ti. Resultados: Emergiram duas categorias da interação aluno-professor que descrevem o processo de aprendizagem. A primeira é "a interação entre os alunos e o professor especialista: um processo de dois", desenvolvido por meio de explicações, intervenções espontâneas dos alunos e exercícios de recapitulação. A segunda trata do "aprendizado: permanece a vida toda", o qual se evidencia na identificação dos diferentes modos de aprender, aprendizagens para aprender e o aprendizado implícito. Conclusões: O processo de aprendizagem na interação entre o professor especialista e os alunos é dinâmico, flexível e aberto. É um espaço onde os alunos e o professor constroem uma relação ativa, proativa e bidirecional que favorece os processos de ensino em Enfermagem, fazendo com que os alunos obtenham aprendizados significativos.

Plalavras-chave: Educação em Enfermagem - Estudantes de Enfermagem - Docentes de Enfermagem; Aprendizagem.

\section{Introducción}

Esta investigación nace del interés en realizar una reconstrucción de sentido mediante la comprensión del proceso de aprendizaje del estudiante de enfermería en su relación con el docente experto. Si bien se conocen las características de esa experticia, poco se ha ahondado en sus potencialidades para el aprendizaje. Además, poseer conocimientos disciplinares no garantiza experticia docente, pues se requieren saberes pedagógicos para organizar situaciones educativas de calidad (1). Se partió del análisis de diversas prácticas pedagógicas, es decir, de las comprensiones, de los conocimientos, de las habilidades y 
de las disposiciones que tienen algunos docentes que desarrollan las clases y las prácticas clínicas en Enfermería. Entre las cuales se destacan aquellas que dan cuenta de una experticia en el rol docente que se puede ver reflejada en mejores aprendizajes. Según García y Montanero (2), una característica que destaca al docente experto es su capacidad de generar un gran número de interacciones que ayuden a los estudiantes a modificar sus errores y a reelaborar sus ideas. El Conocimiento Didáctico del Contenido (CDC) ha sido definido como una forma de acercarse a la experticia de los docentes; en términos de tener la capacidad de integrar su saber disciplinar, es decir, su saber teórico y práctico experiencial con su saber pedagógico, para que sea comprensible, situado y vivencial para los estudiantes (3). Ya se ha demostrado que el CDC contribuye de forma dinámica a las intervenciones que los estudiantes realizan en el aula y a sus repercusiones en el aprendizaje (4).

El análisis de estas prácticas docentes condujo también a reflexionar sobre el papel del estudiante en su propio proceso de formación, ya que el proceso de enseñanza no se puede comprender sin un proceso complementario de aprendizaje. Esta diada supone por lo tanto abordar ambas nociones como dinámicas, independientes y de construcción individual, que a su vez se relacionan y se transforman constantemente (5). Esto llevó al concepto de aprendizaje situado, el cual resalta la interacción social en la adquisición de saberes con sentido para quien aprende (6). Así, el contexto, el lenguaje y las experiencias compartidas cumplen un papel dinamizador en la adquisición de habilidades auténticas (7).

Teniendo en cuenta la naturaleza contextual y social del aprendizaje situado (6), el fenómeno de esta investigación se enmarca en la comprensión de la experiencia de aprendizaje del estudiante de enfermería en la interacción con el docente experto, para contribuir a la reflexión sobre la enseñanza- aprendizaje como partes mutuamente vinculadas e interdependientes.

En la formación en enfermería se encontraron algunas investigaciones sobre el CDC para educar en la comprensión del cuidado (8). En cuanto al aprendizaje se destacan las de corte cuantitativo acerca de los estilos de aprendizaje $(9,10)$ y de incorporación de tecnologías (11). Así mismo, Souza (12), Piña y Aguilar (13) incluyeron la vivencia del estudiante, enfocada al sitio de práctica clínica, en la que resaltaron la reducida intervención del docente clínico a diferencia de su acción en el aula y el laboratorio. Pocos estudios han abordado el aprendizaje desde perspectivas comprensivistas y no se hallaron en Colombia investigaciones en enfermería sobre el aprendizaje situado en concreto.

El presente trabajo refleja la necesidad actual de afrontar los retos del presente, que requieren el análisis y la reflexión sistémica sobre la acción docente-estudiante; conocer la perspectiva del estudiante durante este proceso permitirá tanto a estudiantes como docentes visualizar la particularización de la enseñanza, en el sentido de reconocer y atender sus diferencias individuales (estilos de aprender) como también las formas de enseñar de los docentes (14). Este enfoque de investigación es necesario en la educación en enfermería, ya que ubica en un plano importante la experiencia educativa, para particularizar y personalizar la enseñanza reconociendo las potencialidades y necesidades de los estudiantes (3).

\section{Objetivos}

\section{Objetivo General}

- Describir el proceso de aprendizaje de los estudiantes de enfermería en la interacción con el docente experto

\section{Objetivos específicos}

- Describir la interacción del estudiante de enfermería con el docente experto

- Identificar los aprendizajes que adquiere el estudiante de enfermería en la interacción con el docente experto

\section{Materiales y Métodos}

Esta investigación partió de las siguientes preguntas generales: ¿Cuáles son las características de la relación del estudiante con el docente experto en los espacios educativos?, ¿cuál es el papel del estudiante con relación a la práctica del docente experto?, ¿qué tan diversas son las formas de aprendizaje de los estudiantes?.

Para su desarrollo se llevó a cabo un enfoque cualitativo etnográfico (15), tanto en la recolección de los datos como en el análisis, utilizando herramientas tales como la observación participante, la entrevista think aloud y entrevistas a profundidad. Esto con la intención de interpretar 
el proceso de aprendizaje de los estudiantes en la interacción con el docente experto, como un fenómeno de la vida social al interior del aula de clase (16); y de esta manera llegar a la descripción de los aprendizajes de los estudiantes, desde su perspectiva (17). El acceso al campo se realizó solicitando el aval a una institución formadora en enfermería. En esta institución se seleccionó un "docente experto" reconocido por la comunidad académica por su rigurosidad en la preparación y dominio de los contenidos, por su compromiso con la mejora e innovación de la enseñanza universitaria y por los buenos resultados de aprendizaje en sus alumnos. Se realizó una reunión con el docente experto, quien consultó a los estudiantes para autorizar la observación con video grabación de las clases. Este docente firmó un consentimiento informado.

El trabajo de campo se desarrolló en dos fases metodológicas. En la primera fase se hicieron observaciones participantes (17) durante dos clases (ambas desarrollaron el tema de fisiología cardíaca con el mismo profesor en dos semestres diferentes) para identificar las interacciones entre el docente experto y los estudiantes. Éstas duraron aproximadamente cuatro horas cada una. De ellas, resultaron dos grabaciones en dos planos cada una: uno registrando al profesor y otro a los estudiantes. Las cámaras y los micrófonos estuvieron ubicadas en puntos estratégicos del aula para evitar que los participantes modificaran su conducta. Además, de las notas de campo, se llevó un "registro" (hora, actor, tema) de los momentos en que se realizaban interacciones verbales a través de preguntas, respuestas, comentarios e inquietudes de los estudiantes. Una vez se tuvo el video de las grabaciones de las clases con los dos planos en la pantalla, se procedió a identificar con ayuda del "registro" aquellos momentos en los que se habían dado preguntas o intervenciones espontáneas del estudiante y la consecuente respuesta del docente. Se identificaron 23 momentos que se denominaron episodios, 13 de la primera clase y 10 de la segunda.

En esta primera fase, se realizaron 23 entrevistas Think Aloud, de aproximadamente 15 minutos cada una, a seis estudiantes (tres de la primera clase y tres de la segunda). La entrevista think aloud consiste originalmente en invitar a los individuos a verbalizar sus pensamientos, mientras desarrollan sus acciones (18). En esta investigación se aplicó un protocolo retrospectivo 72 horas después de la clase, en el que cada estudiante evocó el recuerdo sobre la acción (intervención durante la clase) motivado por un video de sí mismo (16).
Es decir, se le presentó el video del episodio que protagonizaba, para que respondiera preguntas tales como: ¿Qué pretendías preguntar? ¿Crees que el profesor entendió tu pregunta? ¿Entendiste la respuesta del profesor? ¿El profesor dio respuesta a tus inquietudes? ¿Qué repercusión tiene para tu aprendizaje? Las entrevistas think aloud, fueron grabadas y transcritas en su totalidad.

En la segunda fase se realizaron las entrevistas en profundidad (17), en las que se destacó la pregunta ¿Cómo la interacción con el docente experto contribuye a tu aprendizaje? Estas entrevistas estuvieron orientadas a comprender los aspectos que componen el proceso de aprendizaje, que involucraran asuntos teóricos, estéticos, sociales, didácticos, metodológicos, éticos, comunicativos y reflexivos (19). Para esto, se siguieron las recomendaciones sobre el muestreo teórico con el fin de profundizar en los datos durante las nuevas entrevistas (20). En total se realizaron 8 entrevistas a 6 estudiantes. Solo dos de ellos habían participado en las entrevistas think aloud. Los criterios para la selección de los estudiantes fueron: Ser estudiante de la Facultad de Enfermería y tener, al menos, una interacción con el profesor seleccionado durante la clase (intercambio de preguntas o incidentes críticos). Se excluyeron aquellos estudiantes que no estuvieran matriculados en el curso.

El análisis fue guiado por los planteamientos de Coffey y Atkinson (21) sobre la codificación. Mediante actividades de teorización y procedimientos analíticos, se identificaron unidades de análisis para su posterior agrupación en conceptos. La comparación, contrastación, agregación y ordenación ayudaron a clasificar y darle orden a los conceptos para la construcción de las categorías. Emergieron aproximadamente 938 unidades de análisis agrupadas en dos categorías logrando la saturación de datos, mediante vínculos y relaciones entre conceptos. Para el análisis se utilizó el programa informático Atlas Ti y se realizaron los memos analíticos.

Este estudio fue aprobado por el Comité de Ética de Investigación de la Facultad de Enfermería de la Universidad de Antioquia en acta No. CEI-FE 2018-37, del 9 de octubre de 2018. Se fundamentó en lo establecido en el Código de Nuremberg (22), en la Declaración de Helsinki (23), en los requisitos éticos de Ezequiel J. Emanuel (24), y en la Resolución 8430 de 1993 del Ministerio de Salud de Colombia (25). De la Ley 911 del 2004 sobre la Responsabilidad deontológica para el ejercicio de la profesión de Enfermería en Colombia (26), se tuvo en cuenta la relación entre 
docentes y estudiantes, la responsabilidad en la investigación y los derechos de autor. Los entrevistados firmaron un consentimiento informado. Para la grabación de las clases, se realizaron consentimientos orales que quedaron registrados en los vídeos. Se garantizó la participación voluntaria y se veló por mantener una actitud respetuosa en una relación de mutua confianza con los participantes como interlocutores válidos.

\section{Resultados}

Se presentan dos categorías que describen el proceso de aprendizaje de los estudiantes de este estudio en la interacción con el docente experto. Estas son: La interacción entre los estudiantes y el docente experto: un proceso de los dos; y los aprendizajes: se quedan para la vida.

\section{Interacciones entre los estudiantes y el docente experto: un proceso de los dos.}

De acuerdo con las observaciones, el aula de clase está llena de interacciones entre el docente y los estudiantes que suponen un esfuerzo mutuo en el que ambas partes ponen su energía para que se generen los aprendizajes de la asignatura: "yo pienso que es de los dos, él hace cosas para que nosotros podamos entender mejor todo, pero nosotros hacemos otras como cuando estudiamos con las preguntas, yo pienso que es de los dos" (A4D5P7). En este estudio las interacciones se presentaron a través de tres mediadores pedagógicos, que son la explicación, las intervenciones espontáneas de los estudiantes y los ejercicios de recapitulación. En los cuales se destaca el humor, la paciencia y la dedicación del docente durante las clases; y se evidencia el interés, el compromiso y una actitud activa por parte de los estudiantes en favor de sus propios procesos de aprendizaje.

\section{La explicación.}

La estrategia que el docente utiliza con frecuencia, según las observaciones, es la clase magistral. En esta, las diapositivas soportan la explicación del tema. La explicación fue una de las interacciones más frecuentes y relevantes entre el docente experto y los estudiantes de este estudio: "Yo no me baso mucho en lo que él escribe si no en lo que explica con respecto a las diapositivas" (A1TE1P1). Es tan importante que "hay varios de esos grupos que graban la voz del profesor" (A2TE12P2), pues "con el audio y con las diapositivas si uno tiene alguna duda se devuelve y escucha la explicación" (A1TE1P1).
De la explicación, los estudiantes destacan acciones del docente en pro de la comprensión del tema, ya que "...el profe explica y a él no le importa si le tiene que explicar 40 veces o buscar 800 millones de ejemplos para que uno entien$d a$ " (A7D4P5). Esta explicación no se limita sólo a la presentación de las diapositivas, pues además “...el docente realiza dibujos en el tablero antes $y$ durante la explicación" (Observación). Según los estudiantes, el docente utiliza otras estrategias para complementar la explicación buscando una comprensión tanto individual como grupal pues él "no le explica igual a todos" (A1TE61), es decir, explica de forma general y también brinda explicaciones para "reafirmar lo que le falta a ciertos estudiantes en especifico" (A1TE61).

Entre estas estrategias, destacan los ejemplos, las anécdotas y las demostraciones. Respecto a los primeros, el profesor utiliza ejemplos simples para explicaciones complejas, es decir, "con algo tan sencillo como una puerta nos explicaba el funcionamiento de las válvulas del corazón" (A1TE6P1). En otras palabras, "él hacía relaciones un poquito bobas pero entendibles. Por ejemplo en el caso del sistema nervioso él ponía una imagen de un grupo de salchichas y nos decía - las más hundidas son como los surcos-" (A2P3P4). Estos ejemplos se constituyen en favorecedores de los aprendizajes porque facilitan la elaboración de los apuntes o notas de clase: "uno escribe palabras claves que sin los ejemplos no sabría qué es, pero con los ejemplos uno se acuerda del funcionamiento, se acuerda de todo" (A1TE6P1). Los participantes reconocen que los ejemplos, son formas de asociar y relacionar que utiliza el docente para que los aspectos teóricos sean comprendidos de una forma más sencilla y didáctica, pues "él hace mucha asociación porque él con nosotros empezaba 'bueno esta es la casa', osteomuscular era la casa, el sistema nervioso es todo el cableado de red, entonces todo el cableado de agua es renal, así lo iba asociando para que uno encontrara como una relación." (A7D4P5).

El docente también utiliza las anécdotas para apoyar sus explicaciones. Durante la observación se identificaron narraciones relacionadas con su experiencia laboral y otras, incluso con vivencias personales. Las diversas formas de explicar suponen un esfuerzo intencional del docente para responder a las necesidades de aprendizaje de los estudiantes: "y otras veces hacía ejemplos totalmente diferentes, lo asocia a lo cotidiano, entonces, se mueve entre muchas formas de explicar. Lo que hace que poquito a poco, el profesor pue- 
da ver la forma que mejor le puede ir para responderle a la pregunta" (A1TE61). Así mismo el docente hace uso de la demostración, es decir, realiza acciones para familiarizar a los estudiantes con términos, conceptos, mecanismos, procesos y demás contenidos complejos y abstractos del curso. Estas demostraciones se realizan en contextos de humor y cercanía, vínculo que genera una dinámica efectiva según los participantes. El profesor realiza demostraciones usando objetos de la clase, dibujos, gráficas, su propio cuerpo o las personas presentes en la clase, para ilustrar algo con referencia al componente teórico y al mismo tiempo hacerlos parte activa del desarrollo de la clase:

"el profesor me dijo 'venga yo lo marco' y... 'quitese la camisa'. Yo me quite la camisa y me paré ahí. Cuando empezó a rayarme y era riéndose y yo volteé y lo miré: 'bueno, hágale profe, ráyame'. Y él' ¿acaso le tengo que pedir permiso?' y yo 'ah bueno profe hágale tranquilo'. Pero es porque uno se toma como esa confianza y eso no lo hace para molestarlo a uno sino para hacer más ameno todo y explicar todo mejor" (A2TE13P).

En general las estrategias que el docente utiliza para ampliar la explicación son pertinentes para la comprensión del tema y su articulación con clases anteriores: “...y luego hizo que asociara temas ya vistos con lo que estaban viendo en el momento, explicándonos por qué el inotropismo del corazón influye en la hipertensión arterial. Entonces sí fue muy pertinente el ejemplo que él nos puso" (A1TE8P3).

\section{Las intervenciones espontáneas de los estu- diantes.}

Otro mediador del aprendizaje que refleja claramente la interacción entre estudiantes y el docente experto está relacionado con las intervenciones espontáneas de los estudiantes. La intervención espontánea más usual es la pregunta, la cual se hace bien sea porque se tienen dudas sobre el tema, es decir, porque "...no haya sido capaz de coger el hilo tan fácil con la primera explicación" (A3D2P1); o también porque se desea corroborar la comprensión del tema: "hay personas que preguntan ya sabiendo las respuestas, como por el hecho de confirmar" (A3D2P1). Las afirmaciones son la otra forma de intervención espontánea. Los estudiantes parafrasean lo explicado por el profesor para también corroborar la comprensión del tema: "Allá quería como llegar, yo lo utilizo para decirme a mi mismo que sí entendí, entonces hay partes en las que él está explicando algo y yo me devuelvo un poquito para decir las cosas con mis propias palabras y que él me diga que estoy en lo correcto, ese aval nos sirve mucho, tanto como para la clase, como para después" (A1TE9P1).

Las intervenciones espontáneas reflejan el ambiente de confianza y tranquilidad que el profesor permite en el aula: "es que soy tímida para preguntar en clase, primero le preguntamos a la compañera y ella me decía que le preguntara al profesor, eso me motivaba y ya entre preguntas se fue creando la confianza y ya después le preguntaba tranquila" (A4D5P7). Ante las inquietudes o afirmaciones, el docente responde de manera adecuada a las necesidades de aprendizaje de los estudiantes. En primer lugar, cuando están equivocados los corrige: "nosotros tenemos una metodología que es prácticamente decir afirmaciones mientras estamos en la clase y si él escucha una que no es o una que puede ayudar a comprender el tema, la coge y vea: 'esto puede ser por esto o lo que dijo usted, así no es" (A1TE9P2). En segundo lugar porque el docente trata de identificar el momento adecuado de la clase para resolver o aclarar la duda: "...en varias ocasiones nosotros hacíamos preguntas sueltas y él decía 'no, espere' o 'enseguida, no se me adelante', entonces termina de explicar y luego sigue '... retomando la pregunta' y explica” (A2TE13P1). Y en tercer lugar porque el docente busca la manera de dar una explicación para todo el grupo, no sólo para el estudiante que hace la pregunta de forma individual. De esta manera, da una respuesta general e incluso, según los estudiantes, el docente intenta responder cada pregunta de diferentes formas acorde con los diferentes estilos de aprendizaje de quienes están en la clase:

"creo que él busca la manera de que todos entiendan, entonces él explica de una manera general con la que la mayoría es capaz de entender y tiene otras formas de explicar cómo más desmenuzado, de explicar el punto como tal, para reafirmar lo que le falta a ciertos estudiantes en específico y creo que tiene como esa forma de entender a los estudiantes y de saber cómo entiende cada uno, porque lo que yo he logrado notar es que no le explica igual a todos" (A1TE61).

\section{Los ejercicios de recapitulación.}

Otro de los mediadores pedagógicos que los estudiantes destacan en la labor del docente experto, tiene que ver con ejercicios de recapitulaciones a través de la lúdica. Mediante estos el profesor valora la comprensión de temas anteriores, al mismo tiempo que aclara las inquietudes y corrige los errores detectados. Por una parte, el desarrollo de los casos clínicos durante la clase, pone en

Rev. cienc. cuidad. 2020;17(3):61-74 
contexto los elementos teóricos y articula el saber previo; mediante análisis en diferentes niveles y discusiones en conjunto: "de una forma más continua, más lúdica, es súper chévere porque a uno le queda claro que sí le sirven las cosas que uno aprende" (A3D2P1). Adicionalmente estos casos ayudan al estudiante a percatarse de su aprendizaje, "ver esa retroalimentación completa de todos los que somos capaces de deducir a partir lo que vimos en la materia, es genial" (A3D2P1).

Por otra parte, los ejercicios de recapitulación a través del juego les permite autoevaluarse al mismo tiempo que adquieren o refinan sus aprendizajes en un ambiente de lúdica: "comenzábamos la clase para ver si habíamos repasado, si nos había quedado claro. Él cogía el kahoot. Entonces, 'ah bueno cojan los celulares'. Eso era chévere porque era cierto tipo de recoch a porque uno jugaba, se distraía y se reía... pero también él aclaraba cuando salían los resultados. Eso era muy bacano porque también es un método para reforzar los conocimientos de las clases anteriores" (A7D4P5). Estas interacciones se presentan como oportunidades de mejora durante su proceso de formación en un ambiente de tranquilidad: "son 4 opciones que te dan y solamente una es la correcta. Entonces, uno dice 'ay tengo que estudiar más eso'. No era evaluativo, era una recocha como para mirar en que estábamos bien y que debiamos mejorar" (A6D3P4). Además, algunos estudiantes resaltan beneficios de esta estrategia relacionados con la coevaluación con sus compañeros de clase "competimos entre nosotros mismos (...) uno ahí por dentro -no, yo le tengo que ganar a todos- "(A3D2P1), otros estudiantes toman estos ejercicios como métodos de autoevaluación, mencionando que "es una forma de reforzar los conocimientos de las clases anteriores" (A6D3P4)

Finalmente, se debe destacar que el humor del docente marca un ambiente de confianza y relajación que propicia el aprendizaje "hace ciertos comentarios que lo hacen a uno reír, le da uno como paso a que las clases de la tarde no sean tan pesadas" (A1TE3P2). También utiliza metáforas jocosas para que el estudiante establezca relaciones o profundice en la temática: "por ejemplo con digestivo, tenemos un chiste que es el efecto morcilla, que es de apretar. Con digestivo el esófago tiene dos movimientos y en el examen estaban estas dos opciones con los que yo lo relacionaba y así me acorde por lo del chiste del efecto morcilla" (A5D1P2).

\section{Los aprendizajes: se quedan para la vida.}

Los estudiantes definen el aprendizaje como un https://doi.org/10.22463/17949831.2269 "conjunto de experiencias de las que se saca algo" (A3D2P1); o como "algo que puede servir para después, ya sea una experiencia negativa o positiva, para desenvolverse más fácil en el futuro si se vuelve a presentar". El aprendizaje también es aquello que sirve para el resto de la vida "yo considero que el aprendizaje es como de verdad interiorizar, pues que te queda para la vida y no solamente de memoria" (A6D3P4). En este sentido, los estudiantes reconocen que con el docente experto superan el aprendizaje memorístico: "las metodologías que él usa se prestan para aprender algo que uno de verdad comprende" (A7D4P5).

La interacción con el docente experto, no sólo contribuye a la comprensión del tema o los temas en particular, sino que dada la diversidad de metodologías utilizadas en las clases, el estudiante se acerca al conocimiento y a la identificación de sus propios estilos y formas de aprender tanto en lo académico, como lo profesional y lo personal "El aprendizaje no es solamente teórico, pues para mi es un conjunto de experiencias, de cosas que uno vive y que saca algo de ellas, y a sean errores, para no repetirlos o cosas nuevas para aplicarlas. Es la formación de lo que somos, de las personas que somos y de lo que queremos ser, pues de lo que uno aprende va entendiendo las cosas diferentes y le puede servir a uno como guía para darse cuenta de qué está haciendo bien, qué está haciendo mal, qué tiene que mejorar, qué tiene que eliminar, entonces el aprendizaje es la herramienta básica para nosotros crecer como personas" (A3D2P1).

Es por lo anterior que estos aprendizajes se dividen en tres apartados: la identificación de las diversas formas de aprender, aprendizajes para aprender y los aprendizajes implícitos.

\section{Identificación de las diversas formas de apren- der.}

Se observaron diversas formas de aprender en la interacción con el docente experto. La mayoría de los estudiantes expresan "yo soy muy gráfico" (A1TE1P3), pues se acuerdan de los contenidos por las imágenes y las gráficas, y mencionan evocar el recuerdo de las diapositivas y apuntes cuando van a responder a una pregunta o una evaluación.

"Yo me acuerdo de la imagen, a veces sí me toca relacionar un poquito porque se me olvidan las cosas, pero se me viene la imagen y de una me acuerdo, por eso también me gusta estudiar de tantas cosas gráficas (A6D3P4).

"Yo por ejemplo en los parciales me acuerdo de
ISSN-PRINT

1794-9831

E-ISSN 2322-7028

Vol. 17 No. 3

Sep - Dic 2020

Cúcuta, Colombia 
la diapositiva y empiezo a repasarla de arriba a abajo y encuentro lo que necesito, lo mismo con mis apuntes". (A3D2P1).

$1794-9831$

E-ISSN 2322-7028

Vol. 17 No. 3

Sep - Dic 2020

Cúcuta, Colombia

Los estudiantes expresan aprender más de lo práctico que de lo teórico, entendiendo por práctico los elementos como "el análisis, la integración" (A5D1P2) y "la familiarización" (A2TE12P1). "Yo aprendo más de lo práctico que de lo teórico, me gusta el análisis de las cosas no solo lo teórico, me gusta más integrar y tenerlo para mí no como tenerlo en un papel (A5D1P2). Otra forma de aprender es la curiosidad: "yo entiendo por curiosidad" (A2TE13P1). Es decir, tienen interrogantes permanentes: "Toda la vida he sido una persona muy curiosa, siempre he tenido dudas, ¿por qué suceden las cosas? ¿qué pasa si cambio esto? ¿qué de esto es anormal?" (A2TE13P1). Como efecto de esta curiosidad uno de los participantes reconoce haberse anticipado a algunos temas: "cuando nosotros vimos el término anastomosis, yo ya sabía qué era. También hablamos del término fibrilación, ya sabía qué era, inotropismo y cronotropismo (...) y es por curiosidad que uno entiende eso" (A2TE13P1).

Así mismo, los estudiantes identifican las diferentes formas de aprender de los compañeros:

"Todo el mundo aprende de diferente forma, entonces como a nosotros se nos da bien charlar y familiarizar más las cosas, (...) hay personas que necesitan pura teoría, lo que a nosotros nos serviría, a ellos no" (A2TE12P1). Incluso, diferencian los estilos y estrategias de aprendizaje propios con los de sus compañeros: "el compañero es muy bueno separándolo todo en una especie de mapa conceptual, Lucas es muy bueno explicando las cosas y también toma buenos apuntes, lo que soy yo me gusta más como el preguntarme, la curiosidad, indagar de por qué se hace esto" (A1TE1P2).

Acorde a las formas de aprender, los estudiantes mencionan estrategias de estudio que favorecen el aprendizaje, tales como los apuntes, la lectura y la escritura, las grabaciones y las asociaciones con otras materias, con la vida cotidiana, con experiencias y conceptos. Tomar notas en clase sirve porque ayuda a recordar lo que el profesor dice, algunos estudiantes escriben palabras clave para recordar los ejemplos y hacen flechas para relacionar información importante "La escritura es muy importante, lo que yo escribo me recuerda cosas que el profesor dice como los ejemplos (A1TE1021). "mi cuaderno está lleno de flechas hacia un lado que dicen palabras (...) yo veo esas flechas y me acuerdo que ahi hay cierta información clave que yo necesito aprenderme, y se me hace muchísimo más fácil recordar las cosas" (A3D2P1).

La lectura y la escritura posteriores a la clase sirven para dar claridad a los temas, como ejemplo, una estudiante menciona que leía mucho un autor pues "a mí renal me quedo súper claro con este autor y mientras que en la clase no tanto. Entonces del libro y del cuaderno hago resumen $y$ estudio de él" (A6D3P4). Otra estudiante expresa que "hay cosas que no se memorizan [fácilmente] y que son importantes de memorizar, por lo que hago resúmenes para recordar toda la información" (A7D4P5). Existen también estrategias auditivas, mediante la grabación de las clases para afinar los apuntes. Con ellas también se reviven las clases "yo personalmente grabo todas las clases, me rio de los chistes otra vez y revivir la clase, la grabo para poder tomar nota después, porque tal vez el profesor sí habla demasiado rápido (...) con las grabaciones yo otra vez me las disfrutaba" (A4D5P7).

\section{Aprendizajes para aprender.}

En la interacción con el docente experto se potencian o emergen unos aprendizajes, relacionados con las capacidades que adquiere o desarrolla el estudiante para acercarse de forma comprensiva y analítica a los contenidos del curso. La asociación es una estrategia para el aprendizaje, mediante la identificación de elementos comunes entre los nuevos contenidos con los conocimientos previos:

"yo quería darle a la bomba sodio potasio una similitud, algo que es un concepto que creo que comprendi muy bien, lo de la retroalimentación negativa entonces no era porque creyera que la bomba sodio potasio era un sistema de retroalimentación sino que podría ser una forma de trabajo que era similar"(A2TE7P1).

Según los estudiantes, la capacidad de asociar su nuevo aprendizaje con la vida cotidiana, con otros cursos, con experiencias previas y con otros conceptos que ya sabían, les ha permitido comprender de manera diferente los temas del curso "pues por ejemplo morfo fisiología y farmacología buscan integrarse, entonces uno entiende, 'ah esto es lo que pasa en el cuerpo. Por eso es que tal medicamento no, tal medicamento si'", (A7D4P5).

Se ha identificado que algunos estudiantes buscan aprender apoyándose en la capacidad de relacionar algún tema de la clase con las vivencias de la cotidianidad, desde saberes previos o desde un vocabulario simple para que el profesor reafirme esta relación. "yo le entendí a él (docente) que Rev. cienc. cuidad. 2020;17(3):61-74 
una célula hace un proceso y le envía la señal a la otra y que ella tiene que hacer lo mismo y por eso lo asocié con eso: 'como yo ya lo hice hágalo usted' (A1TE73). También, cuando el docente describe la Maniobra de Valsalva para explicar un fenómeno del electrocardiograma, un estudiante pregunta " si eso es lo mismo que hacen con la bolsa" (A1TE92). Evocando el recuerdo del por qué había hecho la pregunta, el estudiante explica "yo asimile más que todo en el momento en el que utiliza el aire al soplar con la bolsa, cuando sopla el paciente asmático o una persona que esté hiperventilando. Así que me nació la duda... 'iserá que es la misma maniobra que utilizan las personas para regularse también en la frecuencia cardiaca?" (A1TE92)

Otros estudiantes estimulan su proceso de aprendizaje a partir de experiencias previas para encontrar explicaciones "de algo que pudo haber afectado a un familiar" (A2TE10P2). "Cuando vimos todo lo que era de endocrino, era chévere porque: 'ah bueno, por eso mi mamá vive con sueño'. Uno ya le encuentra una relación en la vida cotidiana" (A7D4P5).

\section{Aprendizajes implícitos.}

En la interacción con el docente experto, los estudiantes adquieren aprendizajes que no están directamente relacionados con el contenido del curso. Se trata de lo que el profesor comunica de manera implícita. Los entrevistados resaltan la pasión y el amor que el docente muestra en su labor: "El amor que demuestra en lo que hace, (...) El nos quiere transmitir que lo que aprendamos, que lo acojamos con amor" (A1TE83). Esa actitud del profesor se expresa en seguridad y alegría por estar estudiando enfermería; despierta en ellos el amor por la docencia y el deseo de ayudar a otros, marcando los horizontes de sus vidas profesionales:

“Él transmite (...) seguridad, alegría por lo que uno está estudiando. Asi puedo terminar yo, con esa emoción, con ese conocimiento, con tantas experiencias. Uno queda con esa expectativa de que ese puedo ser yo" (A5D1P2).

"El profesor además de lo que enseña en la materia, me inspira a querer también enseñar, porque parece muy bacana la metodología que utiliza (...). Además de enseñarme lo de la materia, me enseña una manera adecuada para transmitir ese conocimiento que yo tengo a los demás" (A1TE3P3).

Finalmente, los estudiantes aprenden a equivocarse, porque el docente no sólo les permite cometer errores en un ambiente de confianza, sino que cada error es aprovechado para profundizar en el tema:

"A mí me ha generado mucha confianza las clases con ese profesor, porque cuando uno tiene errores el profesor no lo toma de mala manera sino que explica a uno enfocado en la confusión. Explica más enfocado en eso y no en el tema como tal, sino en lo puntual y esa puntualidad que le explica da pie para que uno comprenda todo" (A1TE92)

\section{Discusión}

Esta investigación se ubica en el espacio que se traza en la interacción del docente y el estudiante. El cual se mueve en un sentido bidireccional que relaciona las acciones del estudiante como: Asociar, analizar, relacionar, concluir, indagar, afirmar y auto evaluar con las del docente, como: explicar, aclarar, evaluar, recapitular, direccionar. Estas acciones se mueven de forma dinámica y profunda para un proceso de adaptación en contexto para los dos actores y para cada uno de sus fines. Esto es semejante a lo que dice Escobar (27) frente a la profunda cooperación y comunicación entre el estudiante y el maestro, pues ante un descuido, el docente sólo podría aspirar a una relación trivial que no repercutirá en aprendizajes significativos. A diferencia de Escobar (27) quien desconoce la participación activa del estudiante para los aprendizajes significativos, esta investigación analiza que los estilos de enseñanza y los estilos de aprendizaje confluyen en el espacio educativo en el que se relacionan el docente experto y los estudiantes. Se destaca como hallazgo la capacidad que tiene el docente de conocer a sus estudiantes y adecuar su estilo de enseñanza a sus formas y necesidades particulares de aprendizaje; así como los resultados positivos de esta capacidad en los aprendizajes de los estudiantes. Al respecto Matagira et al (28), afirman que el conocimiento por parte del docente de los estilos de aprendizaje de los estudiantes es determinante en el uso de los recursos y de los métodos de enseñanza para potenciar la capacidad de aprendizaje del estudiante.

La búsqueda de esta capacidad del docente se encuentra evidenciada en el estudio de Rojas et. al (29), el cual se preocupó por realizar procesos de enseñanza centrados en el estudiante, indagando a los estudiantes sobre su preferencia de estilos de aprendizaje y estilos de enseñanza. Tal como se muestra en lo que expresan los estudian- 
tes de esta investigación, aquél estudio reveló que cada estudiante tiene diferentes necesidades y estilos de aprendizaje que afectan la forma en cómo adquieren los conocimientos. Este hallazgo es importante porque podría prevenir eventuales dificultades de aprendizaje. No obstante, esta investigación difiere en sus conclusiones, al inferir que los estudiantes pueden estar influenciados hacia los estilos de enseñanza de sus docentes, argumentando que los estudiantes tienen mejores logros académicos en la medida en que se inclinen por los estilos de aprendizaje potenciados por sus docentes (29).

Este estudio mostró que cuando el docente reconoce las diferentes formas de aprender de los estudiantes y las necesidades de cada uno, también diseña, moldea o transforma sus estrategias educativas, es decir, que activa su capacidad de reflexión, poniendo toda la atención y el estado de alerta frente al otro (el estudiante) y al propio entorno; lo que supone configurarlos y reinterpretarlos de forma permanente (30). Esa capacidad permite asegurar la comprensión del contenido teórico y el desarrollo de la metacognición, la cual es una capacidad psíquica superior que se traduce en la habilidad de regular, conocer, organizar, monitorear y evaluar las propias potencialidades (31). Según Escobar (27) eso se logra cuando el docente experto es flexible y utiliza el juego y el caso integrativo, cuando realiza un seguimiento personalizado, acompañando la construcción del aprendizaje, en una relación que se establece entre los dos actores y que permite que el estudiante realice procesos mentales más profundos como el análisis, la asociación y la integración del conocimiento teórico; ya sea a su vida personal o al utilizar estos conocimientos previamente adquiridos en las siguientes asignaturas, así como en el ciclo de formación profesional.

Se reconoce que el docente experto genera estrategias motivacionales, ante las cuales el estudiante responde positivamente. Por una parte, les genera confianza para equivocarse lo cual es favorable pues se ha demostrado que una relación pedagógica que ofrece seguridad, empoderamiento, verdad y libertad al estudiante se convierte en una práctica que forma en la autonomía (32). Por otra parte, como se menciona en los hallazgos, los ejercicios de recapitulación son realizados a modo de repaso como parte del proceso de aprendizaje en el aula y se constituyen en procesos de autoevaluación y coevaluación novedosos. Estos son realizados varias veces, mediante metodologías activas y amigables en las cuales los estudiantes participan como sujetos protagonistas de su propio aprendizaje. Al respecto, Moreno (33) propone el término "evaluación interactiva" como una perspectiva reciente en la que se percibe a la evaluación como un elemento integrado al proceso de enseñanza-aprendizaje. Además es un concepto contrario a la evaluación como el producto final. Según el autor, la evaluación debe ser entendida como un proceso natural que utiliza múltiples recursos y no necesariamente un sistema de procedimientos formales. Para ello, es indispensable la comunicación y el conocimiento de los estudiantes, en un proceso que requiere "una atención consciente y reflexiva por parte de los profesores, incorporando el interés como una más de las tareas de la enseñanza" (33).

A propósito de esta iniciativa respecto a las relaciones entre estudiantes y profesores, el autor recomienda que "sean más abiertas en el proceso evaluativo, el cual se considera que debe ser asumido de forma democrática, horizontal y participativa, de modo que la evaluación se convierta en una tarea conjunta entre alumnos y profesores" (33). Por lo tanto, se puede afirmar que la práctica del docente experto demuestra una inclinación actitudinal y práctica hacia los procesos de evaluación interactiva; actitud ha influido en la manera en que los estudiantes participan y perciben la evaluación.

En esta misma línea, la evaluación relacionada con los ejercicios de recapitulación es vista como un proceso que presenta indudables ventajas desde la perspectiva del proceso didáctico, ya que el docente lleva al estudiante a una reflexión sobre su formación y sobre los conocimientos que está obteniendo para la vida. Conclusión similar a la que llegaron Zaragozaet al. (34), cuando describen la evaluación formativa y las ventajas asociadas a la mayor asimilación y relevancia de los aprendizajes. La mayor interacción profesor alumno y por tanto, su vinculación directa con el desarrollo de las competencias profesionales, permiten reconocer la aparición de procesos de coevaluación y autoevaluación asociados a la diversificación de los instrumentos de evaluación utilizados por el docente experto. Estos no están encaminados en la calificación numérica, aunque se ven reflejados al final en la mejora del rendimiento escolar. Al igual que Zaragoza et al. (34), esta investigación establece que utilizar agrupaciones colaborativas, preguntas y actividades que cambien las dinámicas de clase son una forma de utilizar progresivamente la auto-evaluación y la coevaluación.

En este punto es indudable el reconocimiento de ambas partes, tanto de los estudiantes como del 
docente experto, en la dinámica del proceso de enseñanza aprendizaje. Los estudiantes reconocen que si bien los docentes fomentan en ellos acciones de aprendizaje, también ellos contribuyen desde su papel en este aspecto. Según Mercado (5), la enseñanza y el aprendizaje son entendidas como dinámicas independientes y de construcción individual, que a su vez se relacionan y se transforman constantemente, dejando ver lo dinámico del papel del estudiante en ese proceso en el que tienen lugar la asociación, la interpretación y el análisis. Para el caso de esta investigación esto se evidenció por medio de la interacción con el docente experto. De la misma forma en la que Castillo (35) habla de comprensión de las dimensiones fundamentales de las prácticas pedagógicas de las formadoras expertas de enfermería, las profesoras expertas de enfermería dan la voz a los estudiantes para que sean ellos quienes expresen, pregunten, respondan y opinen en las diversas situaciones formativas que enfrentan, realizando una construcción activa del conocimiento, más allá de una pasiva recepción de información.

En este sentido se debe reconocer que la base del éxito en la formación en enfermería es la relación pedagógica pues esta es la que fortalece los rasgos característicos del cuidado de enfermería, ya que contribuye al desarrollo de habilidades interpersonales, éticas y humanas con los estudiantes (36). Esto se traduce en que por medio de las intervenciones en las que se afirman, asocian o concluyen aspectos de la clase o en la realización de preguntas que integran los saberes previos y nuevos, los estudiantes potencian su activa participación en las experiencias formativas.

\section{Conclusiones}

ISSN-PRINT

1794-9831

E-ISSN 2322-7028

Vol. 17 No. 3

Sep - Dic 2020

Cúcuta, Colombia cual se da en un contexto educativo caracterizado por ser un campo social en permanente tensión, en el que se desarrolla el aprendizaje situado. Los actores de este entorno recrean el espacio mediante sus interacciones, donde los estudiantes y el docente construyen una relación activa, propositiva y significativa; en la que se evidencia una dinámica de confianza manifestada por la comunicación horizontal, el lenguaje cotidiano, el lenguaje corporal y el humor que permiten la tranquilidad de los actores que participan en la clase.

- En el proceso de aprendizaje de los estudiantes participantes de este estudio, la interacción estudiante-docente experto favorece el aprendizaje de los estudiantes. Esta interacción es potencializada por mediadores que utiliza el docente, tales como las explicaciones y los ejercicios de recapitulación, que se conectan a través de las intervenciones de los estudiantes con sus concepciones, estilos y estrategias de aprendizaje, que tienen como resultado algunos aprendizajes implícitos, por medio de los cuales se producen los aprendizajes significativos.

\section{Conflicto de Intereses}

Los autores declaran no tener ningun conflicto de intereses.

\section{Referencias bibliográficas}

1. Almonacid-Fierro AA, Merellano-Navarro E, Feu-Molina S, Vizuete-Carrizosa M, Orellana-Fernández R. Perspectiva Cualitativa en la construcción del conocimiento didáctico del contenido del profesorado de Educación Física. Retos [Internet]. 2019 [consultado 3 de abril de 2020]; (36). Disponible en: https://recyt.fecyt.es/index.php/retos/article/view/68840

2. García-García G, Montanero-Fernández M. Comunicación verbal y actividad conjunta en el aula de apoyo. Un análisis comparativo entre profesores expertos y principiantes. Revista Española de Pedagogía [Internet]. 2004 [Consultado 14 de mayo de 2018]; 62(229). Disponible en: https://dialnet.unirioja.es/servlet/articulo? codigo $=1051464$

3. Medina-Moya JL, Rivera-Álvarez LN, Díaz-Álvarez JC. Interpretación docente frente a las contribuciones de los estudiantes en el ámbito universitario. Revista Pedagogía y Saberes [Internet] 2019 [Consultado 3 de abril de 2020]; (51). Disponible en: https://doi.org/10.17227/ 
Juliana Jaramillo-Vargas, Natalia Ossa-Garcés, Marcela Carrillo-Pineda.

pys.num51-8195

ISSN-PRINT

$1794-9831$

E-ISSN 2322-7028

Vol. 17 No. 3

Sep - Dic 2020 Cúcuta, Colombia

4. Medina-Moya JL, Jarauta Borrasca B. Enseñanza y aprendizaje en la Educación Superior. España: Síntesis Editores; 2013

5. Mercado P. Aportes de los Enfoques Socioculturales para "recentrar" los procesos de aprendizaje trabajando en clave dialógica y situacional con la enseñanza. Praxis Educativa [Internet]. 2015 [Consultado 10 de Marzo de 2020]; 19(3). Disponible en: https://cerac.unlpam.edu.ar/ index.php/praxis/article/view/1079/1313

6. Tiburcio-Esteban C, Jiménez-Naranjo Y. La enseñanza del tutunakú a través del aprendizaje situado, bilingüe y dialógico. Revista de Investigación Educativa [Internet]. 2016 [Consultado 21 de mayo de 2018]; (23). Disponible en: http://www.scielo.org.mx/pdf/cpue/n23/18705308-cpue-23-00121.pdf

7. Gómez-Gómez JE, Hernández VL, Morales MA. Arquitectura interactiva como soporte al aprendizaje situado en la enseñanza de la ingeniería. Revista Educación en Ingeniería [Internet]. 2015 [Consultado 30 de mayo de 2018]; 10(20). Disponible en: https://educacioneningenieria.org/index.php/edi/article/view/575

8. Ruiz-Carrillo E, Méndez-García V, Cruz-González J. Sequential discourse analysis of a nursing practice using SDIS-GSEQ: the teacher-student relationship. Revista de Investigación Educativa [Internet]. 2017 [Consultado 30 de mayo de 2018]; (24). Disponible en: http:// www.scielo.org.mx/scielo.php?script $=$ sci arttext\&pid $=\mathrm{S} 1870-53082017000100154 \& 1 \mathrm{~L}-$ $\mathrm{g}=$ es\&tlng=en

9. Agama-Sarabia Adiel, Crespo-Knopfler S. Modelo constructivista y tradicional: influencia sobre el aprendizaje, estructuración del conocimiento y motivación en alumnos de enfermería. Index de Enfermería [Internet]. 2016 [Consultado 14 de mayo de 2018]; 25(1-2). Disponible en: $\underline{h t t p: / / s c i e l o . i s c i i i . e s / s c i e l o . p h p ? s c r i p t=s c i ~ a r t t e x t \& p i d=S 1132-12962016000100025 \& l-~}$ $\underline{\text { ng}}=\mathrm{es} \& \mathrm{tlng}=\mathrm{en}$

10. González-Garza B, Hernández-Castañón MA, Castrejón-Reyes V. Estilos de aprendizaje para el desarrollo de competencias en estudiantes de la licenciatura en enfermería. RIDE Revista Iberoamericana para la investigación y el desarrollo educativo [Internet]. 2018 [Consultado 26de mayo de 2018]; 8(16):351-369. Disponible en: https://doi.org/10.23913/ride.v8i16.345

11. Salazar-Mera J, Tamami-Dávila C. La realidad aumentada y el proceso de enseñanza-aprendizaje de Anatomía en los estudiantes de la carrera de Enfermería de la Facultad de Ciencias de la Salud de la Universidad Técnica de Ambato. [Repositorio de internet] Ecuador: Universidad Técnica de Ambato; 2017 [Consultado 26 de mayo de 2018]. Disponible en: http:// repositorio.uta.edu.ec/handle/123456789/25471

12. Souza D, Schubert Backes VM, Delacanal-Lazzari D. Posibilidades en la educación en enfermería: errar, crear y arriesgar. Enfermería Universitaria [Internet]. 2017 [Consultado 25 de mayo de 2018]; 14 (2). Disponible en: http://dx.doi.org/10.1016/j.reu.2017.02.005

13. Piña-Jiménez I, Amador-Aguilar R. La enseñanza de la enfermería con simuladores, consideraciones teórico-pedagógicas para perfilar un modelo didáctico. Enfermería Universitaria [Internet]. 2015 [Consultado 17 de mayo de 2018]; 12(3). Disponible en: https://dx.doi.org/10.1016/j.reu.2015.04.007

14. Martínez-Royert JC. Prácticas evaluativas del proceso de atención de enfermería: Una visión de docentes y estudiantes. Revista Cuidarte [Internet]. 2017 [Consultado 14 de mayo de 2018]; 8(1). Disponible en: http://dx.doi.org/10.15649/cuidarte.v8i1.351

15. Romero-Quesada MA, Hernández-Quintana AR. El método etnográfico y su relación con el análisis de dominio. Biblios [Internet]. 2015 [Consultado 3 de abril de 2020]; 61. Disponible en: https://doi.org/10.5195/biblios.2015.250

16. Medina-Moya JL, Rivera-Álvarez LN, Rojas-Reyes J. Etnografía de la comunicación en la educación en enfermería: perspectiva metodológica. Revista Cultura de los Cuidados. [Internet]. 2019 [Consultado 3 de abril de 2020] 53. Disponible en: http://rua.ua.es/dspace/hand- 
le/10045/91831

17. Taylor SJ, Bodgan R. Introducción a los métodos de investigación cualitativa. Barcelona: Paidós; 2010.

18. Erickson Anders K. Capturing Expert Thought with Protocol Analysis: Verbalizations of Thinking during Experts' Performance on Representative Tasks. En: Ericson Anders K, Hoffman RR, Kozbelt A, Williams AM. The Cambridge handbook of expertise and expert performance. United Kingdom: Cambridge University Press. Segunda Edición; 2018. P.192-210. https://psycnet.apa.org/doi/10.1017/9781316480748.012

19. López-Vargas B, Basto-Torrado S. Desde las teorías implícitas a la docencia como práctica reflexiva. Educación y Educadores [Internet]. 2010 [Consultado 26 de septiembre de 2018]; 13(2). Disponible en: https://educacionyeducadores.unisabana.edu.co/index.php/eye/article/ view/1699/2258

20. Flick, U. Introducción a la Investigación Cualitativa. España: Morata; 2007.

21. Coffey, A. Atkinson, P. Encontrar sentido a los datos cualitativos: Estrategias complementarias de investigación. Medellín: Editorial Universidad de Antioquia; 2003

22. Código de Nuremberg sobre ética de la investigación en seres humanos. Material de Bioética. Unidad de Humanidades y Ética Médica. Universidad de Navarra [Internet]. Unav.edu. 2007 [consultado 30 de mayo de 2018]. Disponible en: https://www.unav.edu/web/unidad-de-humanidades-y-etica-medica/material-de-bioetica/el-codigo-de-nuremberg

23. Asociación Medica Mundial - The World Medical Association-Declaración de Helsinki de la AMM - Principios éticos para las investigaciones médicas en seres humanos [Internet]. Wma.net. 2017 [consultado 30 de mayo de 2018]. Disponible en: https:/www.wma.net/es/ policies-post/declaracion-de-helsinki-de-la-amm-principios-eticos-para-las-investigaciones-medicas-en-seres-humanos/

24. Suárez-Obando F. Un marco ético amplio para la investigación científica en seres humanos: más allá de los códigos y las declaraciones: la propuesta de Ezekiel J. Emanuel [Internet]. 2015 [consultado 30 de mayo de 2018]; 19(2):182-197. Disponible en: http://dx.doi. org/10.5294/PEBI.2015.19.2.2

25. República de Colombia. Ministerio de Salud. Resolución No 8430 DE 1993, octubre 4, por la cual se establecen las normas científicas, técnicas y administrativas para la investigación en salud. [Internet]. Santa Fe de Bogotá: El Ministerio; 1993 [consultado 30 de mayo de 2018]. Disponible en: https://www.minsalud.gov.co/sites/rid/Lists/BibliotecaDigital/RIDE/DE/DIJ/ RESOLUCION-8430-DE-1993.PDF

26. República de Colombia. Congreso de la Republica. Ley 911 del 2004, octubre 5, por la cual se dictan disposiciones en materia de responsabilidad deontológica para el ejercicio de la profesión de Enfermería en Colombia Santa Fe de Bogotá: Congreso de la Republica; 2004 [consultado 30 de mayo de 2018]. Disponible en: https://www.mineducacion.gov. co/1621/articles-105034_archivo_pdf.pdf

27. Escobar-Medina MB. Influencia de la interacción alumno-docente en el proceso enseñanza-aprendizaje. Revista de Tecnología y Sociedad [Internet]. 2015 [Consultado 16 de marzo de 2020]; 8(5). Disponible en: http://www.udgvirtual.udg.mx/paakat/index.php/paakat/article/view/230/347

28. Matagira-Rondón G, Torres-Panesso MC, Berrio-Osorio N. Estilos de aprendizaje: Un reto curricular para la educación en enfermería. Rev. cienc. cuidad. [Internet]. 2019 [Consultado 3 de abril de 2020]; 16(2). Disponible en: Doi: https://doi.org/10.22463/17949831.1606

29. Rojas-Jara C, Díaz-Larenas C, Vergara-Morales J, Alarcón-Hernández P, Ortiz-Navarrete M. Estilos de enseñanza y estilos de aprendizaje en educación superior: Análisis de las preferencias de estudiantes de Pedagogía en Inglés en tres universidades chilenas. Revista Electrónica Educare [Internet] 2015 [Consultado 14 de mayo de 2018]; 20(3). Disponible en: https:// dx.doi.org/10.15359/ree.20-3.7 
ISSN-PRINT

$1794-9831$

E-ISSN 2322-7028

Vol. 17 No. 3

Sep - Dic 2020

Cúcuta, Colombia
30. Medina-Zuta P. La evaluación formativa desde el rol del docente reflexivo. Maestro y Sociedad. Revista Electrónica para Maestros y Profesores [Internet]. 2019 [Consultada 5 de abril de 2020]; 16(3). Disponible en: https://maestroysociedad.uo.edu.cu/index.php/MyS/article/ view/4979

31. Vargas-Toloza RE. Metacognición, una estrategia de aprendizaje en Enfermería. Revista Ciencia y Cuidado [Internet]. 2018 [Consultado 5 de abril de 2020]; 15(2):5-7. Disponible en: https://doi.org/10.22463/17949831.1397

32. Rivera-Álvarez LN, Medina-Moya JL. The student-nurse pedagogical relationship: a hermeneutic-phenomenological study. Revista Texto context - enferm. [Internet]. 2017 [Consultado 3 de abril de 2020]; 26(2). Disponible en: https://doi.org/10.1590/0104-07072017000560016

33. Moreno-Olivos T. Evaluación del aprendizaje y para el aprendizaje. [Libro en Internet] México: Universidad Autónoma Metropolitana-Cuajimalpa; 2016 [Consultado 14 de marzo de 2020]. Disponible en: https://www.researchgate.net/publication/327824933 Evaluacion del_aprendizaje_y_para_el_aprendizaje_Reinventar_la_evaluacion_en_el_aula

34. Zaragoza-Casterad J, Luis Pascual J, Manrique Arribas J. Experiencias de innovación en docencia universitaria: resultados de la aplicación de sistemas de evaluación formativa. Revista de Docencia Universitaria [Internet]. 2009 [Consultado 14 de marzo de 2020]; 7(4). Disponible en: https://doi.org/10.4995/redu.2009.6232

35. Castillo-Parra S. Práctica pedagógica de profesores/as expertos/as en la formación clínica de estudiantes de enfermería. [Tesis de Internet]. Barcelona: Universidad de Barcelona; 2014. [Consultado 14 de marzo de 2020]. Disponible en: https://www.tdx.cat/bitstream/handle/10803/134821/01.SCP TESIS.pdf?sequence $=1$

36. Rojas-Reyes J, Rivera-Álvarez LN, Morera-Pomarede MJ. Pedagogic Aspects in Nursing Education: Integrative Review. Invest. Educ. Enferm [Internet]. 2018 [Consultado 3 de abril de 2020]; 36(3) Disponible en: https://doi.org/10.17533/udea.iee.v36n3e03 